\title{
Cardio-ankle Vascular Index Is More Closely Associated Than Brachial-ankle Pulse Wave Velocity With Variables Predicting Future Cardiovascular Disease in Patients With Diabetes
}

\section{Taro Saigusa}

Nihon University School of Medicine

Kentaro Watanabe ( $\sim$ watanabe.kentaro@nihon-u.ac.jp )

Nihon University School of Medicine

Yurika Hada

Yamagata University

Kota Ishii

Yamagata University

Wataru Kameda

Yamagata University

Shinji Susa

Yamagata University

Kenichi Ishizawa

Yamagata University

Hisamitsu Ishihara

Nihon University School of Medicine

\section{Research Article}

Keywords: cardio-ankle vascular index, brachial-ankle pulse wave velocity, atherosclerosis, cardiovascular disease risk prediction

Posted Date: September 20th, 2021

DOl: https://doi.org/10.21203/rs.3.rs-876077/v1

License: (c) (i) This work is licensed under a Creative Commons Attribution 4.0 International License.

Read Full License 


\section{Abstract}

Background: This study aimed to compare the usefulness of arterial stiffness parameters, cardio-ankle vascular index (CAVI) and brachial-ankle pulse wave velocity (baPWV), for evaluating future cardiovascular disease (CVD) in subjects with diabetes.

Methods: The study subjects were 277 patients with type 1 or type 2 diabetes. All subjects were evaluated for vascular stiffness using CAVI $(n=154)$ or baPWV $(n=123)$. Carotid intima-media thickness (IMT) and the Suita score were also measured because these are established risk factors for future CVD. Associations of both CAVI and baPWV with these established parameters were evaluated in all subjects, and then in 172 subjects with adjustment for covariates by using propensity score matching.

Results: In all subjects, CAVI and baPWV correlated significantly with both IMT $(r=0.470, P<0.001$, and $r$ $=0.256, P=0.004$, respectively $)$ and the Suita score $(r=0.558, P<0.001$, and $r=0.360, P<0.001$, respectively). The correlation between CAVI and IMT was more significant than that between baPWV and IMT $(Z=2.03, P=0.042)$. Similarly, the correlation between CAVI and the Suita score was more significant than that between baPWV and the Suita score $(Z=2.07, P=0.039)$. After adjustment by matching, significant correlations between CAVI and IMT $(r=0.459, P<0.001)$ and between CAVI and the Suita score $(r=0.526, P<0.001)$ were preserved, though only the association between baPWV and the Suita score was significant $(r=0.270, P=0.011)$ while that between baPWV and IMT showed no significance. Again, CAVI showed a significantly stronger association with the Suita score than baPWV (Z $=1.99, \mathrm{P}=0.046)$.

Conclusions: CAVI is more closely associated than baPWV with variables predicting future CVD in patients with diabetes.

\section{Background}

Subjects with dyslipidemia, hypertension and diabetes mellitus, who smoke, have a high risk of developing cardiovascular disease (CVD) [1]. Furthermore, CVD risk factor clusters reportedly raised CVD risk in a general population cohort [2]. Therefore, evaluating the risk of developing CVD is important for improving CVD mortality in patients with diabetes who have CVD risk factors. Among many tools for CVD risk assessment, brachial-ankle pulse wave velocity (baPWV) [3] and cardio-ankle vascular index (CAVI) [4] are useful and noninvasive. Both baPWV and CAVI evaluate vascular stiffness, which is recognized as a surrogate marker predicting CVD risk [5-8]. The advantages of CAVI and baPWV for evaluating atherosclerosis and for CVD risk assessment remain controversial [9-11]. However, to our knowledge, no studies have compared usefulness for CVD risk assessment between baPWV and CAVI in patients with diabetes.

Thus, we aimed to compare usefulness for cardiovascular risk assessment between baPWV and CAVI in patients with diabetes. We selected carotid intima-media thickness (IMT) and the Suita score for evaluating the risk of CVD development. Carotid IMT is widely recognized as a surrogate marker for the 
risk of CVD in the future $[12,13]$. Additionally, the Suita score provides suitable risk factor categories for predicting the ten-year probability of coronary heart disease (CHD), and is more accurate for predicting $\mathrm{CHD}$ risk than the Framingham risk score in the Japanese population [14].

\section{Methods}

\section{Study subjects}

Two hundred seventy-seven patients with type 1 or type 2 diabetes (173 men and 104 women including 9 type 1 diabetes patients, average age $64.8 \pm 11.5$ years) were recruited as study subjects. All subjects were ambulatory and were followed at the Department of Neurology, Hematology, Metabolism, Endocrinology and Diabetology, Yamagata University Faculty of Medicine and Division of Diabetes and Metabolic Diseases, Department of Internal Medicine, Nihon University School of Medicine. Patients with atrial fibrillation, peripheral arterial disease, malignant diseases, collagen diseases, acute and chronic inflammatory diseases, and/or receiving steroid hormone therapy or other immunosuppressants, were excluded from this study.

\section{Characteristics of study subjects}

We determined clinical characteristics including sex, age, body mass index (BMI), smoking habit, systolic and diastolic blood pressures, anti-hypertensive drug use, statin use, and biochemical variables in all subjects. Biochemical variables, including lipid metabolic parameters, uric acid and $\mathrm{HbA} 1 \mathrm{c}$ were measured after an overnight fast. Low-density lipoprotein (LDL) cholesterol, high-density lipoprotein (HDL) cholesterol, triglycerides, uric acid, creatinine and $\mathrm{HbA} 1 \mathrm{c}$ were measured using an automatic analyzer. The estimated glomerular filtration rate (eGFR) served as an indicator of renal function. eGFR was estimated by the following formula: eGFR $\left(\mathrm{mL} / \mathrm{min} / 1.73 \mathrm{~m}^{2}\right)=194 \times$ Serum creatinine $^{-1.094} \times$ $\mathrm{Age}^{-0.287} \times 0.739$ (if female) [15]. Blood pressure was measured with the patient in a sitting position at the hospital in the morning.

\section{Examination of atherosclerosis and cardiovascular risk}

Carotid IMT, CAVI and baPWV were measured as variables associated with atherosclerosis. Carotid IMT was established as a suitable surrogate marker for the risk of future CVD development [12, 13]. A total of six segments of the near and far walls in the common carotid artery, at the bifurcation, and in the internal carotid artery on the right and left were measured with B-mode imaging of ultrasonography, as described in a previous report [16]. The maximum IMT, including bilateral plaque, was defined as the IMT in all study subjects [16]. Carotid ultrasonographic measurements were performed by experienced clinician. The IMT measurements showed a variability of $8.0 \%$, as previously reported [16].

$\mathrm{CAVI}^{4}$ and baPWV [3] are indicators of arterial stiffness. CAVI is an index of arterial stiffness based on the stiffness parameter $\beta$ [4], while baPWV reflects the stiffness from the aorta to the lower limb arteries [3]. CAVI $[5,6]$ and baPWV $[7,8]$ have been recommended to surrogate markers for CVD. CAVI was measured 
using a Vasera VS-1000 vascular screening system (Fukuda Denshi, Tokyo, Japan). The maximum CAVI on both sides was recorded in each of the study subjects $(n=154)$ enrolled at Yamagata University Hospital and the maximum baPWV measurement was performed using a form PWV/ABI (Omron Healthcare Co., Ltd. Kyoto, Japan) in subjects $(n=123)$ enrolled at Nihon University Itabashi Hospital. CAVI and baPWV in this study were defined as the largest CAVI and baPWV between those of both sides.

For predicting the risk of CVD development, we used the Suita score. The Suita score is an established cardiovascular risk score based on risk factor categories for predicting CHD in the Japanese population [14]. The Suita score consists of the sum of each of these four risk categories and indicates the ten-year probability of $\mathrm{CHD}[14]$.

\section{Statistical analysis}

All subjects were divided into the CAVI $(n=154)$ and baPWV groups $(n=123)$. Furthermore, to adjust for clinical characteristics, we performed one-to-one propensity score matching [17]. Each patient in the CAVI group was paired with a patient in the baPWV group based on the propensity score in this one-to-one matching. Propensity scores were calculated using logistic regression analysis with the covariates of sex, age, BMI, smoking, systolic and diastolic blood pressure, receiving statins and antihypertensive drugs, HDL cholesterol, LDL cholesterol, triglyceride, uric acid, eGFR and HbA1c. Patients with the nearest propensity score within the caliper were paired. A caliper size in the range of 0.20 to 0.25 standard deviation (SD) is recommended $[18,19]$. This study defined a caliper as 0.20 SD. As a result, 86 patients were in both the CAVI and the baPWV group after propensity score matching. The Mann-Whitney $U$ test and the chi-square test were performed to compare clinical characteristics between the CAVI and baPWV groups before or after adjustment by matching. Bonferroni's multiple comparison test was used to compare the mean values of CAVI and baPWV between age groups. Pearson's correlation coefficient and univariate linear regression analysis were used to identify whether CAVI and the Suita score were significantly associated with clinical characteristics, IMT and the Suita score in subjects before or after adjustment by matching. In this univariate linear regression analysis, we assumed CAVI and baPWV to be dependent variables, and sex (men), smoking habit (current), anti-hypertensive drug use or statin use to be independent variables. The observed $Z$ test was used to compare and analyze statistical significance between correlation coefficients. The difference in variance between CAVI and baPWV was evaluated using the F-test of equality of variances. Data are presented as means $\pm S D$, number (\%), coefficient of covariation ( $r$ ), $Z$ values ( $z$ ), F-value or $\beta$ coefficients. A value of $P<0.05$ was considered to indicate statistical significance. All analyses were performed with IBM SPSS Statistics for Windows Version $25 \mathrm{~J}$ (IBM Corp., Armonk, NY, USA).

\section{Results}

\section{Clinical characteristics of study subjects}


The characteristics of the CAVI and baPWV groups before and after matching are shown in Table 1. Sex $(P=0.041)$, age $(P=0.002)$, systolic blood pressure $(P=0.007)$, statin use $(P<0.001)$, LDL cholesterol $(P$ $=0.020)$, eGFR $(P<0.001)$ and $\mathrm{HbA1c}(P<0.001)$ differed significantly between the CAVI and baPWV groups before adjustment by matching (Table 1$)$.

\section{Associations between CAVI or baPWV and clinical characteristics}

Mean values of CAVI and baPWV in all study subjects were 8.69 and $17.74 \mathrm{~m} / \mathrm{sec}$., respectively. Age showed significant associations between CAVI and baPWV both before $(r=0.654$ and 0.494 , respectively, $P<0.001)$ and after $(r=0.632$ and 0.475 , respectively, $P<0.001)$ adjustment by matching (Table 1$)$. CAVI and baPWV rose with age (Fig. 1) in all subjects. Additionally, variations in baPWV in each age group or all subjects were significantly greater those in CAVI (Supplementary Table S1). Adjustment by matching did not change the influence of age on CAVI and baPWV (data not shown).

CAVI showed significant associations with BMI $(r=-0.325, P<0.001)$, systolic blood pressure $(r=0.332, P$ $<0.001)$ and eGFR $(r=-0.243, P=0.002)$ before adjustment by matching. After adjustment, associations of CAVI with BMI $(r=-0.489, P<0.001)$, systolic blood pressure $(r=0.403, P<0.001)$ and eGFR $(r=-0.254$, $P=0.002)$ remained significant, and diastolic blood pressure was also found to show a significant association with CAVI $(r=0.221, P=0.040)$.

baPWV showed significant associations with BMI $(r=-0.197, P=0.029)$, systolic blood pressure $(r=$ $0.339, P<0.001)$, triglyceride $(r=-0.211, P=0.019)$ and eGFR $(r=-0.217, P=0.016)$ before adjustment by matching (Table 2). After adjustment by matching, the associations remained significant for systolic blood pressure $(r=0.382, P<0.001)$ and triglyceride $(r=-0.256, P=0.017)$.

Univariate linear regression analysis indicated significant associations between baPWV and antihypertensive drug use $(\beta=1.547, P=0.029)$ before adjustment by matching. After adjustment by matching, however, current smoking was found to show a significant correlation $(\beta=-1.195, P=0.026)$, while the significant association with antihypertensive drug use was unchanged $(\beta=1.796, P=0.031)$.

\section{Associations of CAVI and baPWV with cardiovascular risk factors}

CAVI and baPWV showed significant correlations with both IMT $(r=0.470, P<0.001$, and $r=0.256, P=$ 0.004, respectively) (Fig. 2A, 2C) and the Suita score ( $r=0.558, P<0.001$, and $r=0.360, P<0.001$, respectively) (Fig. 2B, 2D). The correlations between CAVI and IMT and the Suita score were both more significant than those between baPWV and IMT $(Z=2.03, P=0.042)$ and the Suita score $(Z=2.07, P=$ 0.039).

After adjustment by matching, the significant correlations of CAVI with IMT $(r=0.459, P<0.001)$ (Fig. 3A) and the Suita score $(r=0.526, P<0.001)$ (Fig. 3B) were unchanged, while baPWV was only significantly associated with the Suita score $(r=0.270, P=0.011)$ (Figure 3D). Again, CAVI showed a significantly stronger association with the Suita score than did baPWV $(Z=1.99, P=0.046)$. 


\section{Discussion}

Our results indicate both CAVI and baPWV to be significantly associated with carotid IMT and the Suita score, widely used surrogate variables for assessing atherosclerosis and the risk of developing CVD in the future. Notably, CAVI was found to be more closely associated than baPWV with variables predicting CVD development in patients with diabetes. To our knowledge, this is the first study to demonstrate a difference in clinical significance between CAVI and baPWV in patients with diabetes.

We can speculate as to why CAVI more closely reflects atherosclerosis and CVD development in the future than baPWV in patients with diabetes. One possibility is that peripheral arterial stiffness influences CAVI and baPWV measurements. CAVI reflects central arterial stiffness because the basic formula for calculating CAVI is based on heart-ankle PWV. ${ }^{4} \mathrm{CAVI}$ is more influenced by central arterial stiffness than baPWV because the route of the pulse wave in baPWV reflects the status of both central and peripheral arteries, including those from the aortic annulus to the brachium [20]. Atherosclerosis, renal and cardiac function are reportedly more closely associated with central arterial stiffness (carotid-femoral PWV) than baPWV [21]. Our results also revealed a significant inverse association between CAVI and eGFR before and after adjustment by matching, whereas the significant association between baPWV and eGFR was seen only before adjustment. Furthermore, a recent study of patients with chest pain syndrome showed that carotid IMT had a somewhat more significant association with CAVI than with baPWV [9].

Another factor is that CAVI shows higher reproducibility than baPWV. We found variations in baPWV in each age group as well as in all subjects to be significantly larger than those in CAVI. We thus suggest that the blood pressure at the time of measurement may influence the difference in variation between CAVI and baPWV. baPWV is decreased in association with reductions in systolic and diastolic blood pressures when antihypertensive agents are administered, but CAVI does not change [22]. Furthermore, baPWV showed a significant increase when systolic blood pressure elevated more than $10 \mathrm{mmHg}$, while CAVI showed no significant change [23]. These studies suggested that baPWV is more susceptible than CAVI to changes in blood pressure. Therefore, we speculate that baPWV shows less reproducibility than CAVI due to blood pressure variability at the time of measurement. The blood pressure variability in patients with diabetes is high compared to that in subjects with normal glucose tolerance [24]. In addition, this variability increases with age [25].

The accuracy of the path length formula also influences the variation in both CAVI and baPWV measurements. Magnetic resonance imaging results established that the estimated path length from the heart to the ankle in CAVI well reflects the true path length [26]. Conversely, the arterial path length formula in the baPWV measurement is calculated based on the path length from the aortic annulus to the ankle subtracted from the path length from the aortic annulus to the brachium, and this arterial path length is overestimated because the estimated path length from the aortic annulus to the brachium in the baPWV measurement is shorter than the actual path length [27]. 
This study demonstrated significant associations of systolic blood pressure with both CAVI and baPWV. The latter is closely associated with blood pressure because baPWV depends on blood pressure at the time of measurement [22]. Furthermore, subjects with hypertension had a higher average baPWV than subjects without hypertension [28]. Indeed, we found taking antihypertensive medication to be significantly positively associated with baPWV. The reasons for systolic blood pressure being significantly correlated with CAVI are, however, uncertain. CAVI is independent of blood pressure at the time of measurement because the determination of CAVI is based on measurement of the stiffness parameter $\beta$ that is not influenced by blood pressure [4]. However, CAVI was reported to be significantly, but more weakly than baPWV, correlated with blood pressure in subjects receiving routine health checkups [23]. Moreover, a cross-sectional study in elderly individuals indicated that systolic blood pressure in high-CAVI group (CAVI $\geq 9$ ) patients correlated significantly with $\mathrm{CAVl}$, whereas blood pressure in the low-CAVI (CAVI < 9) group patients showed no significant associations with CAVI [29]. The results of these and our studies allow us to conclude that the CAVI, as well as baPWV, might be influenced by blood pressure.

The elevations of CAVI and baPWV with age have previously been documented [6, 28], and our study also showed age-related increases in CAVI and baPWV. Increasing aortic stiffness with age contributes to the observed increases in CAVI [30] and baPWV [31]. Our results also demonstrated that CAVI and baPWV well reflect increased aortic stiffness with age in patients with diabetes.

We demonstrated CAVI to be inversely associated with BMI before and after adjustment by matching, while baPWV showed an association only before adjustment. Previous cross-sectional studies indicated CAVI and baPWV to correlate negatively with BMI $[32,33]$. The reasons for these inverse associations have yet to be clarified. Body length affects the measurements of both CAVI and baPWV because the formulas for calculating these indices include pulse wave velocity from the heart to the ankle [4] and the brachium to the ankle [3], respectively. BMI is calculated as body weight/height squared, and BMI would thus be expected to have an inverse association with CAVI and baPWV. Additionally, in our study, baPWV showed a negative association with triglyceride. BMI was positively associated with triglyceride (data not shown). It is reasonable to speculate that the positive association between BMI and triglyceride in our study explains the significant inverse association between baPWV and triglyceride before and after adjustment by one-to-one case matching.

The invert association between smoking and baPWV was indicated after adjustment by matching in our study, although acute or chronic smoking causes a significant increase in arterial stiffness [34]. The reasons why smoking was negatively associated with smoking remain unclear. Subjects with smoking habit were younger, and indicated higher prevalence of the use of antihypertensive medication than subjects without (data not shown). We concluded that receiving antihypertensive medication and the age in subjects with smoking habit may cause the invert association between baPWV and smoking habit.

\section{Conclusion}


Our study demonstrated that CAVI is more closely associated than baPWV with the variables predicting the risk for developing CVD in the future in patients with diabetes. Thus, CAVI might be a more suitable tool for cardiovascular risk assessment than baPWV. CAVI appears to be particularly useful for assessing patients with diabetes who show a high risk for developing CVD.

\section{Abbreviations}

CAVl; cardio-ankle vascular index, baPWV; brachial-ankle pulse wave velocity, CVD; cardiovascular disease, IMT; intima-media thickness, CHD; coronary heart disease, BMl; body mass index, LDL; lowdensity lipoprotein, HDL; high-density lipoprotein, eGFR; estimated glomerular filtration rate, SD; standard deviation.

\section{Declarations}

\section{Acknowledgements}

Not applicable.

\section{Author's contribution}

T.S. and K.W. designed the study protocol and drafted the manuscript. K.W., Y.H. and K.I. corrected the clinical data. S.S. and K.I. carried out the literature search and drafted the manuscript. W.K., S.S., H.I., and K.I. reviewed the manuscript.

\section{Funding}

Not applicable.

\section{Availability of data and materials}

Data available on request from the correspondence author (Kentaro Watanabe; watanabe.kentaro@nihonu.ac.jp).

\section{Ethics approval and consent to participate}

The study was designed in accordance with the principles of the Declaration of Helsinki. The study design was cross-sectional and employed a clinical database. This study protocol was approved by the ethics committee of Yamagata University Faculty of Medicine (No. 2021-12) and Nihon University School of Medicine (No. RK-180911-10). The protocol of this study is available on the web page of the Ethics Committee. All potential study subjects were given the opportunity to opt out. The requirement for written informed consent was waived by the ethics committee of Yamagata University Faculty of Medicine and Nihon University School of Medicine due to the retrospective nature of the study. 
Not applicable.

\section{Competing interests}

The authors declare that there is no conflict of interest.

\section{Author details}

${ }^{1}$ Division of Diabetes and Metabolic Diseases, Department of Internal Medicine, Nihon University School of Medicine.

${ }^{2}$ Department of Neurology, Hematology, Metabolism, Endocrinology and Diabetology, Yamagata University Faculty of Medicine.

\section{References}

1. Dawber TR, Kannel WB. The Framingham Study. An epidemiological approach to coronary heart disease. Circulation. 1966;34:553-5.

2. Ninomiya T, Kubo M, Doi Y, Yonemoto K, Tanizaki Y, Rahman M, et al. Impact of metabolic syndrome on the development of cardiovascular disease in a general Japanese population: the Hisayama study. Stroke. 2007;38:2063-9.

3. Munakata M. Brachial-ankle pulse wave velocity in the measurement of arterial stiffness: recent evidence and clinical applications. Cur Hypertens Rev. 2014;10:49-57.

4. Namba T, Masaki N, Takase B, Adachi T. Arterial Stiffness Assessed by Cardio-Ankle Vascular Index. Int J Mol Sci. 2019;20:e366.

5. Gohbara M, Iwahashi N, Sano Y, Akiyama E, Maejima N, Tsukahara K, et al. Clinical impact of the Cardio-Ankle Vascular Index for predicting cardiovascular events after acute coronary syndrome. Circ J. 2016;80:1420-6.

6. Sato $Y$, Nagayama D, Saiki A, Watanabe R, Watanabe $Y$, Imamura $H$, et al. Cardio-Ankle Vascular Index is Independently Associated with Future Cardiovascular Events in Outpatients with Metabolic Disorders. J Atheroscler Thromb. 2016;23:596-605.

7. Ninomiya T, Kojima I, Fukuhara M, Hirakawa Y, Hata J, Kitazono T, et al. Brachial-ankle pulse wave velocity predicts the development of cardiovascular disease in a general Japanese population: the Hisayama Study. J Hypertens. 2013;31:477-83.

8. Turin TC, Kita Y, Rumana N, Takashima N, Kadota A, Matsui K, et al. Brachial-ankle pulse wave velocity predicts all-cause mortality in the general population: findings from the Takashima study, Japan. Hypertens Res. 2010;33:922-5.

9. Takai A, Ogawa H, Wakeyama T, Iwami T, Kimura M, Hadano $\mathrm{Y}$, et al. Cardio-ankle vascular index is superior to brachial-ankle pulse wave velocity as an index of arterial stiffness. Hypertens Res. 2008;31:1347-55. 
10. Satoh H, Shimazu A, Kato Y, Araki R, Koyama K, et al. Evaluation of the cardio-ankle vascular index, a new indicator of arterial stiffness independent of blood pressure, in obesity and metabolic syndrome. Hypertens Res. 2008;31:1921-30.

11. Kato A, Takita T, Furuhashi M, Maruyama Y, Miyakawa H, Kumagai H. Brachial-ankle pulse wave velocity and the cardio-ankle vascular index as a predictor of cardiovascular outcomes in patients on regular hemodialysis. Ther Apher Dial. 2012;16:232-41.

12. Bots ML, Hoes AW, Koudstaal PJ, Hofman A, Grobbee DE. Common carotid intima-media thickness and risk of stroke and myocardial infarction: the Rotterdam Study. Circulation. 1997;96:1432-7.

13. Kitagawa $K$, Hougaku $H$, Yamagami $H$, Hashimoto $H$, Itoh T, Shimizu $Y$, et al. Carotid intima-media thickness and risk of cardiovascular events in high-risk patients. Results of the Osaka Follow-Up Study for Carotid Atherosclerosis 2 (OSACA2 Study). Cerebrovasc Dis. 2007;24:35-42.

14. Nishimura K, Okamura T, Watanabe M, Nakai M, Takegami M, Higashiyama A, et al. Predicting coronary heart disease using risk factor categories for a Japanese urban population, and comparison with the Framingham risk score: the Suita study. J Atheroscler Thromb. 2014;21:78498.

15. Matsuo S, Imai E, Horio M, Yasuda Y, Tomita K, Nitta K, et al. Revised equations for estimated GFR from serum creatinine in Japan. Am J Kidney Dis. 2009;53:982-92.

16. Watanabe K, Suzuki T, Nakano H, Oba K. Usefulness of carotid parameters measured by ultrasonography as a marker of atherothrombotic infarction and lacunar infarction in high-risk elderly people. Geriatr Gerontol Int. 2006;6:87-93.

17. Rosenbaum PR, Rubin DB. The central role of the propensity score in observational studies for causal effects. Biometrika. 1983;70:41-55.

18. Austin PC. Optimal caliper widths for propensity-score matching when estimating differences in means and differences in proportions in observational studies. Pharm Stat. 2011;10:150-61.

19. Rosenbaum PR, Rubin DB. Constructing a control group using multivariate matched sampling methods that incorporate the propensity score. Am Stat. 1985;39:33-8.

20. Sugawara J, Hayashi K, Tanaka $\mathrm{H}$. Arterial path length estimation on brachial-ankle pulse wave velocity: validity of height-based formulas. J Hypertens. 2014;32:881-9.

21. Lu Y, Zhu M, Bai B, Chi C, Yu S, Teliewubai J, et al. Comparison of carotid-femoral and brachial-ankle pulse-wave velocity in association with target organ damage in the community-dwelling elderly Chinese: The Northern Shanghai Study. J Am Heart Assoc. 2017;6:e004168.

22. Shirai K, Utino J, Saiki A, Endo K, Ohira M, Nagayama D, et al. Evaluation of blood pressure control using a new arterial stiffness parameter, cardio-ankle vascular index (CAVI). Curr Hypertens Rev. 2013;9:66-75.

23. Kobozono T, Miyata M, Ueyama K, Nagai A, Yutaka O, Kusano K, et al. Clinical significance and reproducibility of new arterial distensibity index. Circ J. 2007;71:89-94.

24. Joshipura KJ, Muñoz-Torres FJ, Campos M, Rivera-Díaz AD, Zevallos JC. Association between withinvisit systolic blood pressure variability and development of pre-diabetes and diabetes among 
overweight/obese individuals J Hum Hypertens. 2017;32:26-33.

25. Takashima N, Ohkubo T, Miura K, Okayama A, Okuda N, Nakagawa H, et al. Factors associated with intra-individual visit-to-visit variability of blood pressure in four countries: the INTERMAP study. $\mathrm{J}$ Hum Hypertens. 2019;33:229-36.

26. Miyoshi T, Ito H. Assessment of arterial stiffness using the cardio-ankle vascular index. Pulse (Basel). 2016;4:11-23.

27. Sugawara J, Hayashi K, Tanaka H. Arterial path length estimation on brachial-ankle pulse wave velocity: validity of height-based formulas. J Hypertens. 2014;32:881-9.

28. Tomiyama H, Arai T, Koji Y, Yambe M, Motobe K, Zaydun G, et al. The age-related increase in arterial stiffness is augmented in phases according to the severity of hypertension. Hypertens Res. 2004;27:465-70.

29. Roengrit T, Sri-amad R, Huipao N. High systolic blood pressure is associated with increased cardioankle vascular index in elderly. Artery Research. 2021;27:25-31.

30. Sun CK. Cardio-ankle vascular index (CAVI) as an indicator of arterial stiffness. Integr Blood Press Control. 2013;6:27-38.

31. O'Rourke MF, Hashimoto J. Mechanical factors in arterial aging. J Am Coll Cardiol. 2007;50:1-13.

32. Kabutoya T, Hoshide S, Fujiwara T, Negishi K, Nishizawa M, Yamamoto M, et al. Age-related difference of the association of cardiovascularrisk factors with the cardio-ankle vascular index in the Cardiovascular Prognostic Coupling Study in Japan (the Coupling Registry). J Clin Hypertens (Greenwich). 2020;22:1208-15.

33. Gomez-Marcos MA, Gomez-Sanchez L, Patino-Alonso MC, Recio-Rodriguez JI, Gomez-Sanchez M, Rigo $F$, et al. A body shape index and vascular structure and function in Spanish adults (MARK study): A cross-sectional study. Medicine (Baltimore). 2018;97:e13299.

34. Kubozono T, Miyata M, Uemura K, Hamasaki S, Kusano K, Kubozono O, et al. Acuute and chronic effect of smoking on arterial stiffness, Circ J. 2011;75:698-702.

\section{Tables}

Table 1. Clinical characteristics of study subjects. 


\begin{tabular}{|c|c|c|c|c|}
\hline \multirow[b]{2}{*}{ Clinical characteristics } & \multicolumn{2}{|c|}{ Before adjustment by matching } & \multicolumn{2}{|c|}{ After adjustment by matching } \\
\hline & $\begin{array}{l}\text { CAVI group } \\
\quad(\mathrm{n}=154)\end{array}$ & $\begin{array}{l}\text { baPWV group } \\
\quad(\mathrm{n}=123)\end{array}$ & $\begin{array}{l}\text { CAVI group } \\
(\mathrm{n}=87)\end{array}$ & $\begin{array}{c}\text { baPWV group } \\
(\mathrm{n}=87)\end{array}$ \\
\hline Sex (men) & $88(57.1)$ & $85(69.1)^{\#}$ & $52(59.8)$ & $53(60.9)$ \\
\hline Age (years) & $62.9 \pm 12.3$ & $67.0 \pm 10.0^{* * *}$ & $65.1 \pm 12.3$ & $67.0 \pm 10.9$ \\
\hline Body mass index & $24.60 \pm 4.89$ & $24.71 \pm 3.85$ & $24.59 \pm 4.76$ & $24.77 \pm 3.91$ \\
\hline Smoking habit (current) & $34(22.1)$ & $30(24.4)$ & $22(25.3)$ & $16(18.4)$ \\
\hline \multicolumn{5}{|l|}{ Blood pressure (mmHg) } \\
\hline Systolic & $127.0 \pm 16.5$ & $133.0 \pm 18.2^{* *}$ & $130.3 \pm 15.4$ & $132.3 \pm 19.1$ \\
\hline Diastolic & $76.3 \pm 10.9$ & $77.0 \pm 11.7$ & $76.4 \pm 11.2$ & $77.1 \pm 11.9$ \\
\hline Antihypertensive drug use & $93(60.4)$ & $84(68.3)$ & $59(67.8)$ & $56(64.4)$ \\
\hline Statin use & $69(44.8)$ & $81(65.9)^{\# \#}$ & $44(50.6)$ & $52(59.8)$ \\
\hline HDL-CHOL (mmol/L) & $1.42 \pm 0.53$ & $1.42 \pm 0.37$ & $1.48 \pm 0.53$ & $1.45 \pm 0.41$ \\
\hline LDL-CHOL (mmol/L) & $2.77 \pm 0.80$ & $2.57 \pm 0.78^{*}$ & $2.63 \pm 0.69$ & $2.68 \pm 0.80$ \\
\hline Triglyceride $(\mathrm{mmol} / \mathrm{L})$ & $1.62 \pm 1.02$ & $1.42 \pm 0.77$ & $1.34 \pm 0.74$ & $1.41 \pm 0.67$ \\
\hline Uric acid $(\mu \mathrm{mol} / \mathrm{L})$ & $323.1 \pm 90.1$ & $316.6 \pm 70.4$ & $319.3 \pm 81.6$ & $316.3 \pm 70.7$ \\
\hline eGFR (mL/min/1.73 m2) & $83.49 \pm 33.21$ & $69.82 \pm 17.98^{* *}$ & $77.08 \pm 27.46$ & $70.64 \pm 19.65$ \\
\hline HbAlc (\%) & $9.01 \pm 2.41$ & $7.14 \pm 0.84^{* *}$ & $7.66 \pm 1.43$ & $7.26 \pm 0.89$ \\
\hline
\end{tabular}

Mean \pm SD, $n(\%)$

${ }^{\star} \mathrm{P}<0.05,{ }^{* \star} \mathrm{P}<0.01$ vs CAVI group by Kruscal-Wallis test; ${ }^{\#} \mathrm{P}<0.05$ vs CAVI group by Chi-squire test.

HDL, high-density lipoprotein; LDL, low-density lipoprotein; eGFR, estimated glomerular filtration rate, $\mathrm{HbA} 1 \mathrm{c}$, glycosylated hemoglobin.

Table 2. Correlation coefficient between CAVI, baPWV and clinical characteristics. 


\begin{tabular}{lcccc}
\hline \multirow{2}{*}{ Clinical characteristics } & \multicolumn{2}{c}{ Before adjustment by matching } & \multicolumn{2}{c}{ After adjustment by matching } \\
& CAVI $(\mathrm{n}=154)$ & baPWV $(\mathrm{n}=123)$ & CAVI $(\mathrm{n}=87)$ & baPWV $(\mathrm{n}=87)$ \\
\hline Age (years) & $0.654^{* *}$ & $0.494^{* *}$ & $0.632^{* *}$ & $0.475^{* *}$ \\
Body mass index & $-0.325^{* *}$ & $-0.197^{*}$ & $-0.489^{* *}$ & -0.187 \\
Blood pressure $(\mathrm{mmHg})$ & & & & \\
$\quad$ Systolic & $0.332^{* *}$ & $0.339^{* *}$ & $0.403^{* *}$ & $0.382^{* *}$ \\
$\quad$ Diastolic & 0.139 & 0.050 & $0.221^{*}$ & 0.099 \\
HDL-CHOL $(\mathrm{mmol} / \mathrm{L})$ & 0.093 & -0.056 & 0.175 & -0.025 \\
LDL-CHOL $(\mathrm{mmol} / \mathrm{L})$ & -0.085 & -0.087 & 0.004 & -0.181 \\
Triglyceride $(\mathrm{mmol} / \mathrm{L})$ & -0.133 & $-0.211^{*}$ & -0.156 & $-0.256^{*}$ \\
Uric acid $(\mu \mathrm{mol} / \mathrm{L})$ & -0.067 & -0.068 & -0.175 & -0.195 \\
eGFR $\left(\mathrm{mL} / \mathrm{min} / 1.73 \mathrm{~m}^{2}\right)$ & $-0.243^{* *}$ & $-0.217^{*}$ & $-0.254^{*}$ & -0.179 \\
HbAlc $(\%)$ & -0.121 & 0.071 & 0.092 & 0.109 \\
\hline
\end{tabular}

${ }^{*} \mathrm{P}<0.05,{ }^{* *} \mathrm{P}<0.01$.

HDL, high-density lipoprotein; LDL, low-density lipoprotein; eGFR, estimated glomerular filtration rate, HbA1c, glycosylated hemoglobin.

Table 3. Univariate linear regression analysis predicting for association between clinical characteristics, CAVI and baPWV 


\begin{tabular}{|c|c|c|c|c|}
\hline \multirow[b]{2}{*}{ Clinical characteristics } & \multicolumn{2}{|c|}{ Before adjustment by matching } & \multicolumn{2}{|c|}{ After adjustment by matching } \\
\hline & $\begin{array}{l}\text { CAVI group } \\
\quad(\mathrm{n}=154)\end{array}$ & $\begin{array}{l}\text { baPWV group } \\
\quad(\mathrm{n}=123)\end{array}$ & $\begin{array}{l}\text { CAVI group } \\
\quad(\mathrm{n}=87)\end{array}$ & $\begin{array}{c}\text { baPWV group } \\
\quad(\mathrm{n}=87)\end{array}$ \\
\hline Sex (men) & $88(57.1)$ & $85(69.1)^{\#}$ & $52(59.8)$ & $53(60.9)$ \\
\hline Age (years) & $62.9 \pm 12.3$ & $67.0 \pm 10.0^{* * *}$ & $65.1 \pm 12.3$ & $67.0 \pm 10.9$ \\
\hline Body mass index & $24.60 \pm 4.89$ & $24.71 \pm 3.85$ & $24.59 \pm 4.76$ & $24.77 \pm 3.91$ \\
\hline Smoking habit (current) & $34(22.1)$ & $30(24.4)$ & $22(25.3)$ & $16(18.4)$ \\
\hline \multicolumn{5}{|l|}{ Blood pressure $(\mathrm{mmHg})$} \\
\hline Systolic & $127.0 \pm 16.5$ & $133.0 \pm 18.2^{* *}$ & $130.3 \pm 15.4$ & $132.3 \pm 19.1$ \\
\hline Diastolic & $76.3 \pm 10.9$ & $77.0 \pm 11.7$ & $76.4 \pm 11.2$ & $77.1 \pm 11.9$ \\
\hline Antihypertensive drug use & $93(60.4)$ & $84(68.3)$ & $59(67.8)$ & $56(64.4)$ \\
\hline Statin use & $69(44.8)$ & $81(65.9)^{\# \#}$ & $44(50.6)$ & $52(59.8)$ \\
\hline HDL-CHOL $(\mathrm{mmol} / \mathrm{L})$ & $1.42 \pm 0.53$ & $1.42 \pm 0.37$ & $1.48 \pm 0.53$ & $1.45 \pm 0.41$ \\
\hline LDL-CHOL $(\mathrm{mmol} / \mathrm{L})$ & $2.77 \pm 0.80$ & $2.57 \pm 0.78^{*}$ & $2.63 \pm 0.69$ & $2.68 \pm 0.80$ \\
\hline Triglyceride $(\mathrm{mmol} / \mathrm{L})$ & $1.62 \pm 1.02$ & $1.42 \pm 0.77$ & $1.34 \pm 0.74$ & $1.41 \pm 0.67$ \\
\hline Uric acid $(\mu \mathrm{mol} / \mathrm{L})$ & $323.1 \pm 90.1$ & $316.6 \pm 70.4$ & $319.3 \pm 81.6$ & $316.3 \pm 70.7$ \\
\hline eGFR (mL/min/1.73 m2) & $83.49 \pm 33.21$ & $69.82 \pm 17.98^{* *}$ & $77.08 \pm 27.46$ & $70.64 \pm 19.65$ \\
\hline HbAlc (\%) & $9.01 \pm 2.41$ & $7.14 \pm 0.84^{* * k}$ & $7.66 \pm 1.43$ & $7.26 \pm 0.89$ \\
\hline
\end{tabular}

$\beta$ co-efficient (95\% confidence interval).

Dependent variable: CAVI or baPWV, independent variables: sex (men), smoking habit (current), antihypertensive drug use or statin use.

CAVI, cardio-ankle vascular index; baPWV, brachial-ankle pulse wave velocity.

\section{Figures}




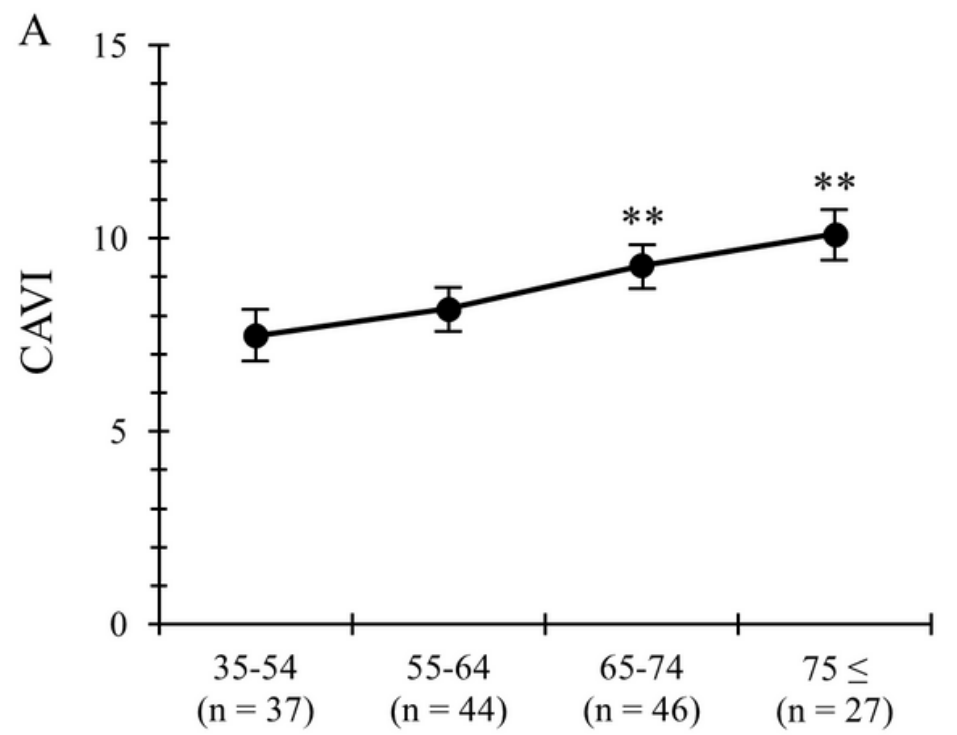

Age (years)

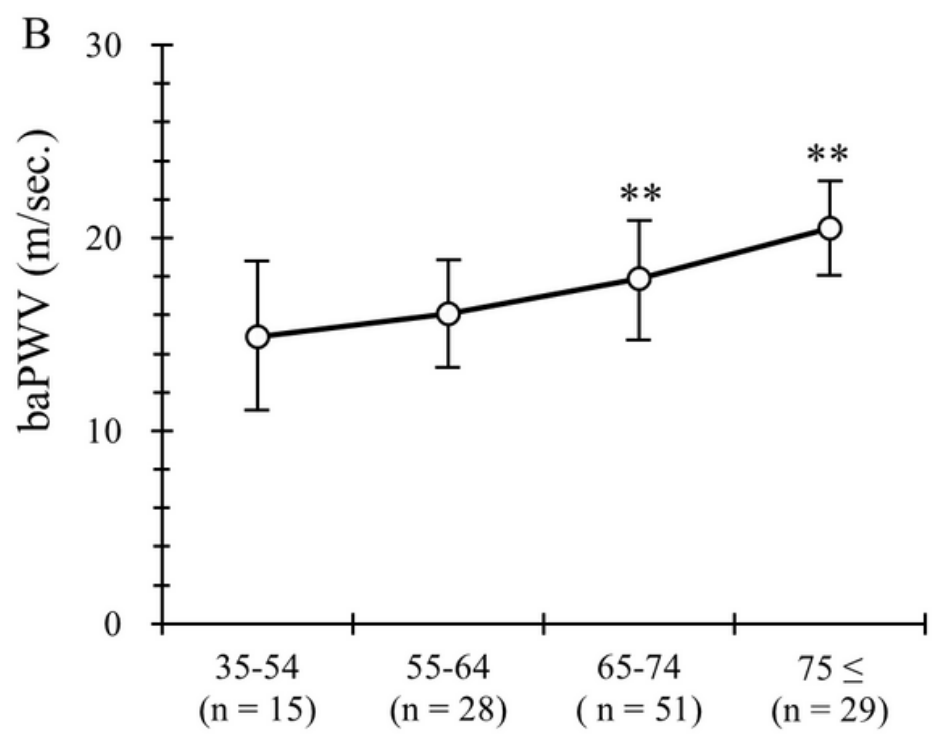

Age (years)

Figure 1

Distributions of CAVI and baPWV by age groups in all subjects. A: CAVI, $B$ : baPWV. ${ }^{\star * P}<0.01$ vs subjects from 35 to 54 years of age. CAVl; cardio-ankle vascular index, baPWV; brachial-ankle pulse wave velocity.
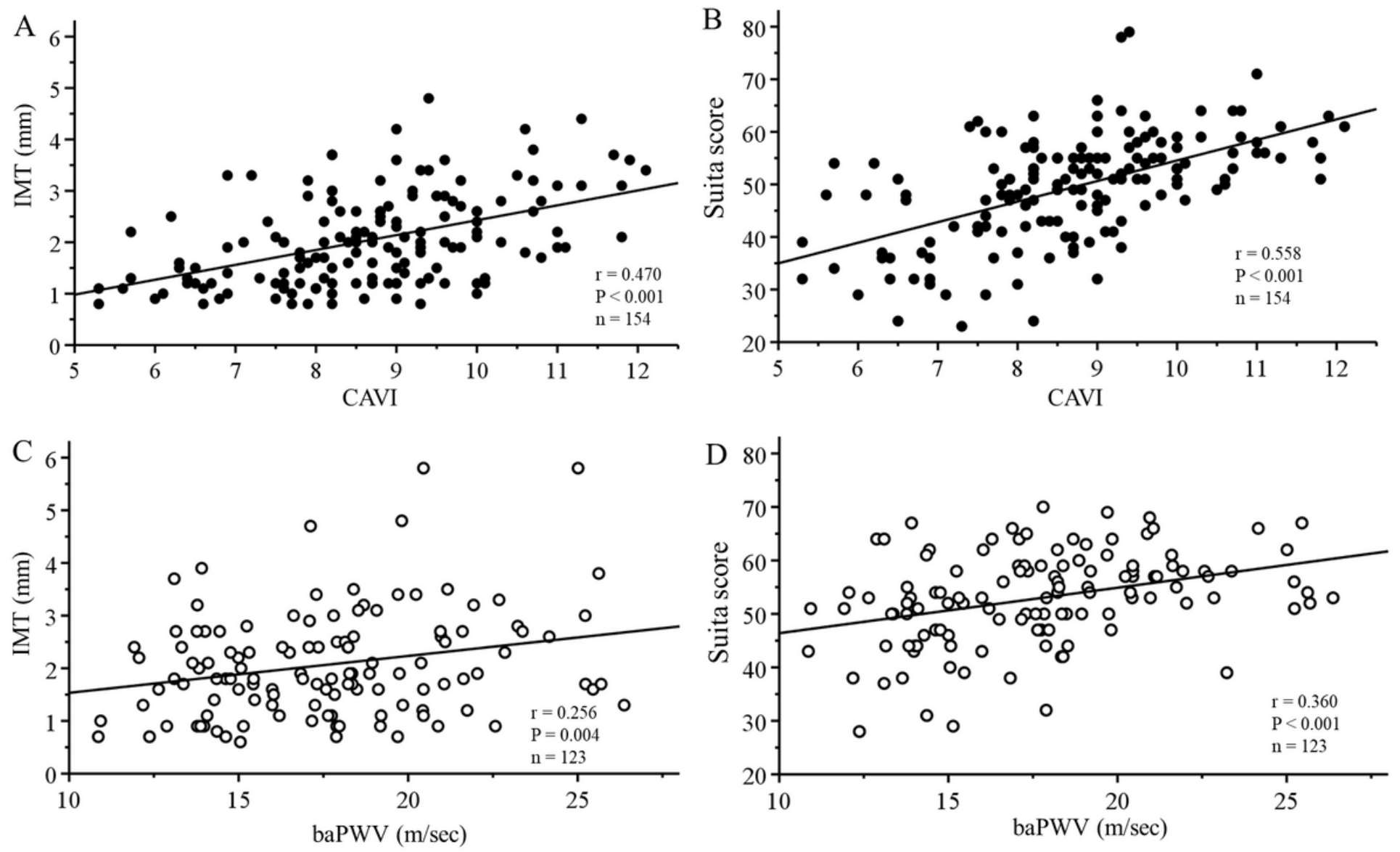

Figure 2 
Correlations of CAVI and baPWV with IMT and the Suita score before adjustment by matching. A: CAVI and IMT, B: CAVI and Suita score, C: baPWV and IMT, D: baPWV and Suita score. CAVl; cardio-ankle vascular index, baPWV; brachial-ankle pulse wave velocity, IMT; intima-media thickness.
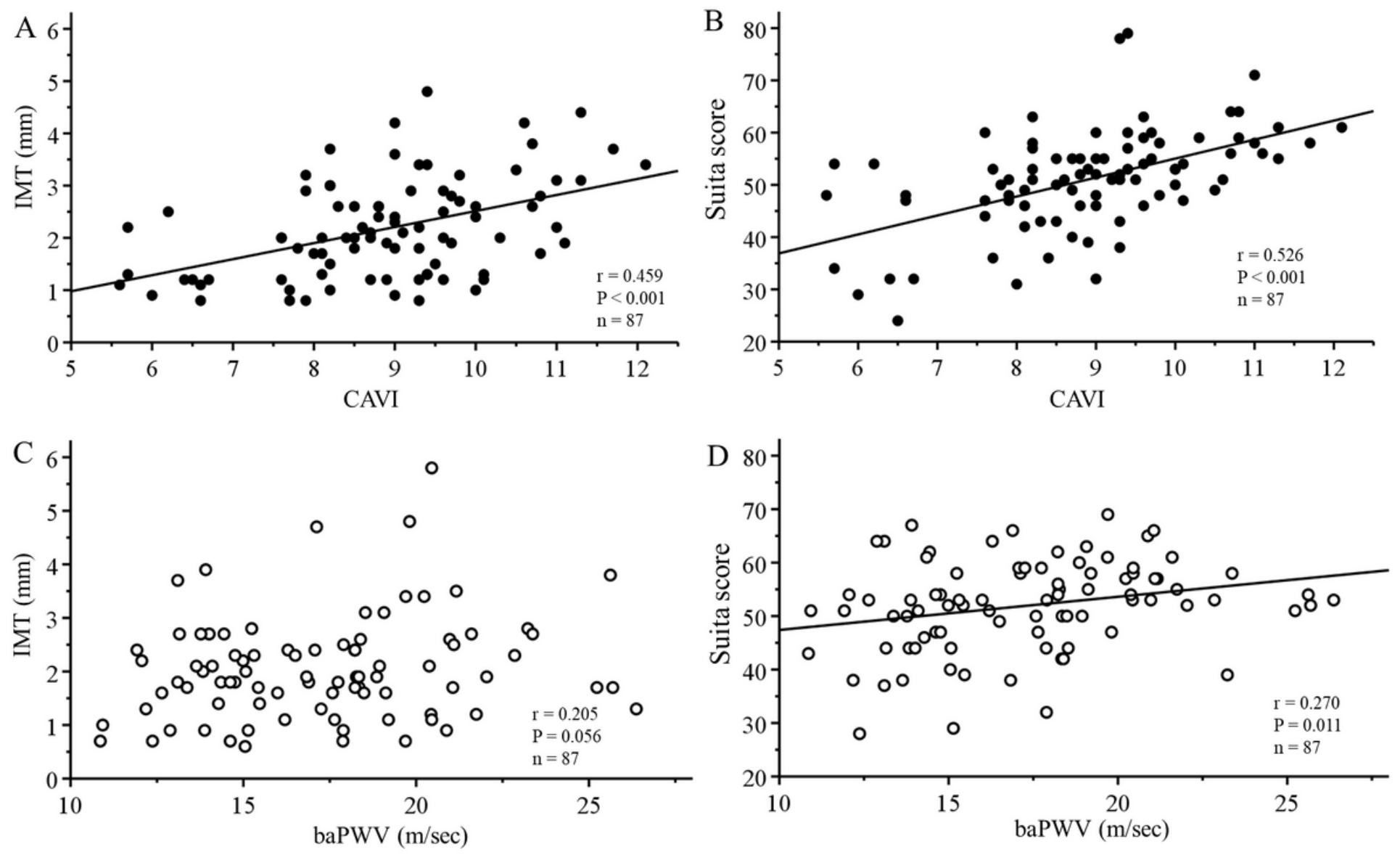

Figure 3

Correlations of CAVI and baPWV with IMT and the Suita score after adjustment by matching. A: CAVI and IMT, B: CAVI and Suita score, C: baPWV and IMT, D: baPWV and Suita score. CAVI; cardio-ankle vascular index, baPWV; brachial-ankle pulse wave velocity, IMT; intima-media thickness.

\section{Supplementary Files}

This is a list of supplementary files associated with this preprint. Click to download.

- SupplementalTablesBMCcardiovasculardisorders.docx 